\title{
An Entity Resolution using Query Sensible Approach
}

\author{
Bheema Rasagna* and Kummaragunta Lakshmi Prasanna \\ Department of CSE, Visvodaya Engineering College, JNTUA, Kavali - 524201, Andhra Pradesh, India: \\ rasagnabheema87@gmail.com, lakshmi.kummaragunta@gmail.com
}

\begin{abstract}
:
Objective: To study the Entity Resolution (ER) using Qualified Security Assessor (QSA) to minimize the pre-processing steps which are required to fetch the data a given Structured Query Language (SQL)-like selection correctly. Methods/ Statistical Analysis: In recent times, the problem of Entity Resolution is carried out in the context of data warehousing as an offline pre-processing step prior to the making data accessible to analysis - an approach that works well under paradigm settings. Such an offline approach, however, is not possible in budding applications that requires analyzing only small portions of the whole dataset and generating answers in (near) real-time. In this we presented an approach named as (QSA) to minimizing the data pre-processing steps in query processing to detect the objects. Findings: To test the efficiency of the QSA we collected the bibliographic data from the Google Scholar. This data contained the top 50 computer related researchers each having h-index of 60 or higher. The dataset contained 16,396 records where $14.3 \%$ are duplicates. Then we apply the QSA using two blocking function to group the data records that might be duplicates together. Finally, we ensure pair wise resolve function to detect weather two records represent the same real world entity or not accurately. Applications/Improvements: We have used the semantics of such a collection predicate to decrease pre-processing in the Entity Resolution. The accuracy of the entity resolution using QSA will be increased.
\end{abstract}

Keywords: Data Wareho use, Entity Resolution, Offline Pre-processing, Query Processing, Rudimentary

\section{Introduction}

This study deals with the problem of Entity Resolution (ER) challenge. Predictably, entity resolution brings out in the background of data warehousing as an offline pre-processing step earlier to building data available to analysis - an approach that facilitates well under idea settings. Such an offline approach is not possible in growing applications that need analyzing only tiny portions of the complete data and the producing answers in real-time ${ }^{1}$.

Our approach is stimulated by various key points of view $^{2}$. First, the requirement for current analysis needs modern environments to achieve timely analytical tasks, building it impractical for those environments to utilize detained hypothesis back-end pre-processing technologies. Second, in the context of data analysis task, where a data analyst may measure and examine data as part of a single step, the method will know "what to clean" only at query time. Last, outlines wherein a little establishment contains an expansive dataset, yet require analyze just small segments of it to answer some efficient inquiries instantly. In such a case, it would be pointless for that organization to use their insufficient accessible assets on pre-handling absolute information, for the most part given that the vast majority of it will be copy.

The earlier methods cannot utilize the rules of such a variety predicate to decrease pre-processing. To deal with these new pre-processing confronts we proposed a QSA to data pre-processing ${ }^{3,4}$. QSA is a fully new balancing model for enhancing the effectiveness: It distinguishes from blocking $\underline{5}$ and is usually additional efficient in collaborating with jamming. QSA measure results are similar

${ }^{*}$ Author for correspondence 
to those obtained by first using a common pre-processing algorithm and then requesting on top of the pre-processed data. Though, in diverse cases QSA measures such results more effectively. A pre-processing step is rudimentary if QSA can promise that it calculates answers without idea about the result of this decision.

These studies discuss significantly our earlier work in various directions. Initially we introduced the concept of rudimentary for an enormous class of SQL collection queries and industrial techniques to classify rudimentary pre-processing steps; we officially enforce the idea of rudimentary in this paper.

Expressly, we explore how the strength of the clustering algorithm ${ }^{6}$ influences the evaluation efficiency of QSA. In our initial work 2 , we developed QSA to work with keen clustering techniques ${ }^{6}$. We simplify QSA to work with lazy clustering techniques in this study. Fourth, we develop new proposals that optimize the processing of equality and range queries.

\section{Research Method}

\subsection{Query Sensible Solution}

In this subdivision, we illustrate our solution named as Query-Sensible Solutions. Here, discuss eager clustering techniques $-\underline{\underline{6}-\underline{10}}$ and also we discuss the lazyQSA, which exploits with lazy with eager clustering techniques.

\subsection{QSA using Eager Clustering Techniques}

The principle mission of QSA is to work out a response to question $\mathrm{Q}$ creatively. The outcome ought to be proportional to influence a standard ER algorithm on the total information and after that asking for the resulting pre-processed information with query $\mathrm{Q}$. In this division, we extend QSA to enforce with eager clustering techniques. Keep in mind that a conventional eager ER algorithm, which utilizes Transitive Closure Clustering to gather matching entities collectively into clusters, operates by iteratively picking a couple of nodes to determine straightaway, at that point applying for the resolve function, combining nodes if the determination gives a positive answer and then iterating the process. Our eagerQSA approach is very comparable to eager-ER with two considerable differences.

Tentatively, eager-QSA has comprised the accompanying advances:
- Creating and Labelling the Graph. The arrangement begins by making and labelling graph $\mathrm{G}$.

- Choosing an Edge to Resolve. Rely upon its edge-choosing strategy; the arrangement picks edge $e_{i j}$ to decide. Instinctively, such an arrangement ought to pick $\mathrm{e}_{i j}$ in a methodology that decides it would endure eager-QSA to likewise expediently add a little group delegates to the outcome gathering or would break many associated cliques. The one that has affirmed the best outcomes are relied upon picking edges with respect to its weight, where weight wij for edge $e_{i j}$ is computed by including the estimations of its event nodes: $w_{i j}=v_{i l} \oplus v_{j l}$.

- Lazy Edge Removal. We have displayed numerous improvements in eager-QSA. The algorithm checks if the chosen edge $\mathrm{e}_{i j}$ still endures in this progression. On the off chance that it isn't, the algorithm will come back to Step 2 to pick one more edge.

Monitor that after compromise of node, just edges that are all inclusive to them must stay in G. In any case, confirming for parallel edges and afterward firmly expelling them from resultant information records at the procedure of the union is an $\mathrm{O}(|\mathrm{R}|)$ strategy in like manner for each merge activity. To deter this cost, eager-QSA does not evacuate the edges at the procedure of the merge, yet disposes of them lethargically in this progression. It does as such in $\mathrm{O}(1)$ time by confirming if $\mathrm{v}_{i}\left(\right.$ or $\left.\mathrm{v}_{j}\right)$ of edge $\mathrm{e}_{i j}$ has been converged with some other hub vk by the calculation on a previous cycle and consequently $(i) v_{i}\left(o r v_{j}\right)$ was eliminated from $\mathrm{V}$ might be or (ii) $\mathrm{v}_{i}$ isn't in $\mathrm{v}_{j}$ 's locale or the other way around.

- Rudimentary Testing. The algorithm, in this progression, expects to leave behind calling decide $e_{i j}$ by confirming on the off chance that it is rudimentary.

- Stopping Condition. If an edge $e_{i j} \in \mathrm{E}$ that is neither decided nor rudimentary, at that point the algorithm iteratively by going to Step 2 .

- Computing the Answer. In this end, the algorithm delivers the quiry's definitive answer utilizing the fundamental outcome rules $\mathrm{S}$.

Therefore, our expected translate into tricky algorithms that use the above advances. Such algorithms ought to consolidate the quantity of notice of the progression decided function and have the capacity to consummately and effectively find an outcome to a given query. In gathering, 
the picked algorithms must themselves be exceptionally profitable, something else, work cost will be expanded, and a basic speculation, for example, deciding all $\mathrm{O}\left(\mathrm{n}^{2}\right)$ edges in uninformed order might be high effective.

Unequivocally, eager-QSA analyzes for rudimentary by utilizing a vague however quick check to decide whether $\mathrm{e}_{i j}$ can conceivably be a piece of any interrelated faction whatsoever. Algorithm 1, can probably be seen as spreads the accompanying advances:

Algorithm 1. Rudimentary-Testing

Input: an edge $e_{i j}$, graph $G$, and a query $Q$

Output: a labeled edge $e_{i j}$

1: if IS-IN-PRESERVING $\left(p, \oplus, a_{l}\right)$ and MIGHT-

CHANGE-ANS $\left(\varnothing, v_{i} \oplus v_{j}, Q\right)$ then

2: res $\leftarrow \mathfrak{R}\left(v_{i}, v_{j}\right)$

3: if res $=$ MustMerge then

4: $A_{\text {cur }} \leftarrow A_{\text {cur }} \cup\left\{v_{i} \oplus v_{j}\right\}$

5: $V_{\text {maybe }} \leftarrow V_{\text {maybe }}-\left\{v_{i}, v_{j}\right\}$

6: else if res $=$ MustSeparate then

7: $E \leftarrow E-\left\{e_{i j}\right\}$

8: else $l\left[e_{i j}\right]=$ maybe

9: else if CHECK-POTENTIAL-CLIQUE $\left(e_{i j}, G, Q\right)$ then

10: res $\leftarrow \mathfrak{K}\left(v_{i}, v_{j}\right)$

11: if res $=$ MustMerge then

12: $v_{i} \leftarrow v_{i} \oplus v_{j}$

13: $\mathcal{N}\left[v_{i}\right]=\mathcal{N}\left[v_{i}\right] \cap \mathcal{N}\left[v_{j}\right]$

14: $V_{\text {maybe }} \leftarrow V_{\text {maybe }}-\left\{v_{j}\right\}$

15: else if res $=$ MustSeparate then

16: $E \leftarrow E-\left\{e_{i j}\right\}$

17: else $l\left[e_{i j}\right]=$ maybe

18: else $E \leftarrow E-\left\{e_{i j}\right\}$

- Edge Minclique Check Optimization. In other streamlining algorithm sends here, a looks at for an unmistakable case enable the calculation to dispense with two nodes from the graph paying little respect to a union, critical to additional investment funds. Explicitly, this surprising case endures when triple $\left(\mathrm{p}, \oplus, \mathrm{a}_{1}\right)$ is in-preserving and edge $\mathrm{e}_{i j}$ without anyone else's input can change the present response to Q. Provided that this is true, at that point $\mathrm{e}_{i j}$ isn't rudimentary and the calculation calls decide on it.

Presently, on the off chance that decide returns MustMerge, the algorithm joins the consolidated node $\left(v_{i} \oplus v_{j}\right)$ to the outcome space and after that disposes of $v_{i}$ and $v_{j}$ nodes from $G$. The algorithm can accomplish this optimization.

- Check for Potential Clique. On the off chance that Step 1 does not make a difference, the algorithm utilize Algorithm 2 to check if $e_{i j}$ can firmly be one of any reasonable clique whatsoever. On the off chance that it returns true, the function on $\mathrm{e}_{i j}$ and labels $\mathrm{G}$ in like manner. In the event that it returns false, the capacity marks $\mathrm{e}_{i j}$ as rudimentary.

The Algorithm 2 consistently checks if an edge $\mathrm{e}_{i j}$ can probably be possessed in a significant/negligible clique by any stretch of the imagination. It is a safe correct capacity: it returns false just when $e_{i j}$ isn't to be a piece of pertinent/ negligible clique.

Algorithm 2 Check-Potential-Clique Input: an edge $e_{i j}$, a graph $G$ and a query $Q$ Output true if $e_{i j}$ is in a potential clique, false otherwise 1: if MIGHT-CHANGE-ANS $\left(\varnothing, v_{i} \oplus v_{j}, Q\right)$ then

2: return true

3: $V_{\text {intersect }} \leftarrow \mathcal{N}\left[v_{i}\right] \cap \mathcal{N}\left[v_{j}\right]$

4: for each $v_{k} \in V_{\text {intersect }}$ do

5: $v_{\text {old }} \leftarrow v_{\text {new }}$

6: $v_{\text {new }} \leftarrow v_{\text {old }} \oplus v_{k}$

7: if MIGHT-CHANGE-ANS $\left(v_{\text {old }}, v_{\text {new }}, Q\right)$ then

8: return true

9: return false

After the algorithm performed managing out edges, it computes its definitive answer $A_{\text {cur }}$ to query $Q$ relies upon the appropriate response semantics $S$ the client requested.

Algorithm 3 starts by summing nodes from $\mathrm{V}$ maybe which assure $\mathrm{Q}$ to $\mathrm{A}_{\text {cur }}$. At this stage $\mathrm{A}_{\text {cur }}$ guarantee answer semantics. As such, $A_{\text {cur }}$ might include copies as well as it probably won't equal to the conventional combined portrayal delivered by eager-ER.

\section{Algorithm 3 Compute-Answer} Input: a current answer $A_{c u r}$, a set of maybe nodes $V_{\text {maybe }}$, a query $Q$, and an answer semantic $S$.

Output: a clean final answer $A_{\text {cur }}$, 
1: for each $v_{i} \in V_{\text {maybe }}$ do

2: if SATISFY - $Q\left(v_{i}, Q\right)$ then

3: $A_{\text {cur }} \leftarrow A_{\text {cur }} \cup v_{i}$

4: if $S=$ Distinct then

5: for each $v_{i} \uparrow A_{c u r}$ do

6: for each $v_{j} \neq v_{i} \in A_{\text {cur }}$ do

7: if $\mathfrak{K}\left(v_{i}, v_{j}\right)=$ MustMerge then

8: $v_{i} \leftarrow v_{i} \oplus v_{j}$

9: $A_{\text {cur }} \leftarrow A_{\text {cur }}-\left\{v_{j}\right\}$

10: else if $S=$ Exact then

11: for each $v_{i} \in A_{c u r}$ do

12: for each $v_{j} \neq v_{i} \in A_{\text {cur }} \cup V_{\text {maybe }}$ do

13: if $\mathfrak{K}\left(v_{i}, v_{j}\right)=$ MustMerge then

14: $v_{i} \leftarrow v_{i} \oplus v_{j}$

15: if $v_{j} \in A_{\text {cur }}$ then

16: $A_{c u r} \leftarrow A_{c u r}-\left\{v_{j}\right\}$

From a speculative perspective, our eager-QSA could be valuable to look at the features of answer precision. Note that on the off chance that the purpose work is always exact, excited ER will ascertain grouping $\mathrm{C}$ that is comparable to the ground-truth bunching $\mathrm{C}_{\mathrm{gt}}$.

\section{Result and Analysis}

In this division, we for all intents and purposes ascertain the adequacy of our QSA techniques on genuine and synthetic data. We think about our QSA answers for different query types. Particularly, we essentially assess eager-QSA with eager-ER as far as the conclusion to-end running time and the quantity of calls to determine. Note that the two methodologies utilize Transitive Closure Clustering to group the organized elements. In addition, we assess lazy-QSA with lazy-ER as far as the quantity of determination summons. Note that the two methodologies utilize a variety of the diminishing irregularity Correlation Clustering to group the coordinated entities. In diagram 1 utilizes a set of GTE $(\geq)$ queries to illustrate the influence of Rudimentary verification by comparing eager-QSA with eager-ER. For each threshold t, we run 50 queries - one query for the each author. Then, we convene the time taken by all these 50 queries and divide it by 50 to report the average running time for every threshold. The best piece of Figure 1 plots the unequivocal end-to-end execution time in spite of the fact that the base part plots the quantity of calls to determine of eager-QSA and eagerER for different estimations of the Government Technical Education (GTE) question threshold t. Figure 2 depicts
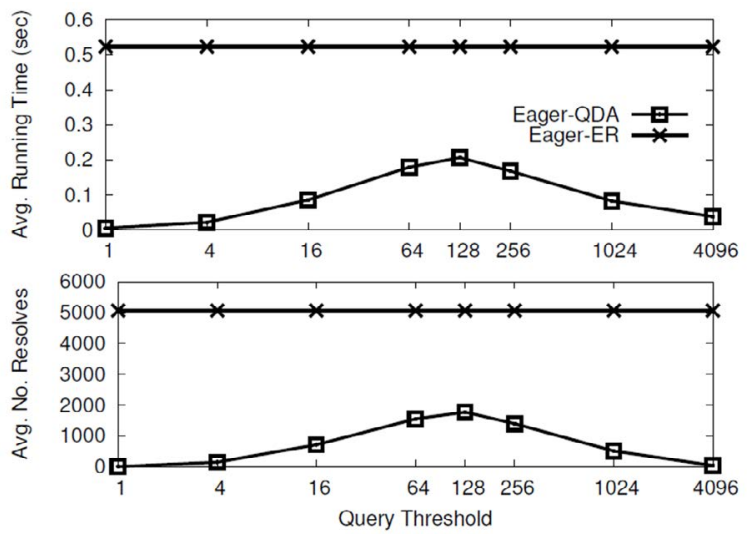

Figure 1. Eager-QSA vs. eager-ER.
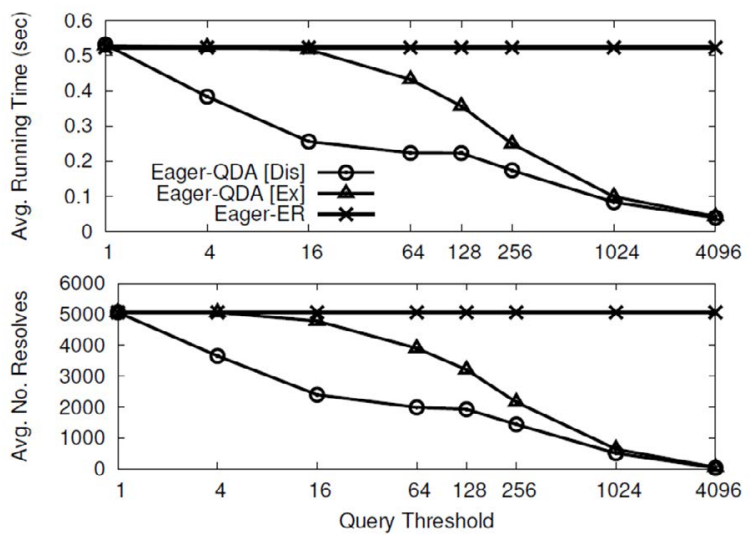

Figure 2. Answer semantics.

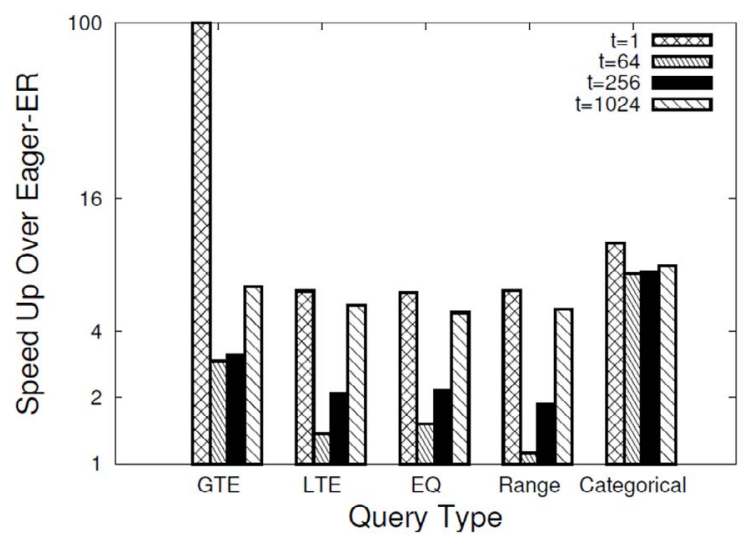

Figure 3. Speed up of eager-QSA. 
the conclusion to-end running time and the quantity of purposes called by eager-QSA misusing unique and precise answer semantics. Figure 3 depicts the speed up of eager-QSA over eager-ER for 5 distinct query types utilizing 4 distinct threshold values. The eager-QSA efficient over eager-ER is measured as the laterally running time of eager-ER divided by that of eager-QSA.

\section{Conclusion}

In this study, we have contemplated the query-sensible ER difficulty in which information is pre-processed "on-thefly" in the points of view of a selection query. We have developed QSA, which adequately issues the insignificant number of pre-processing steps required to absolutely answer a given determination query. We formalized the issue of Query-sensible ER and indicated exactly how distinct pre-processing steps can be abbreviated. This exploration offers path to a few fascinating bearings for further examination.

\section{References}

1. Cohen W. A comparison of string distance metrics for name-matching tasks. II Web International Conference on Information Integration on the Web; 2003. p. 73-8.

2. Chen Z. Exploiting context analysis for combining multiple entity resolution systems. SIGMOD International
Conference on Management of data; 2009. p. 207-18. https://doi.org/10.1145/1559845.1559869

3. Altwaijry H. Query: A framework for integrating entity resolution with query processing. International Conference on Very Large Data Bases (VLDB); 2015. p. 120-31. https:// doi.org/10.14778/2850583.2850587

4. Ananthakrishna R. Eliminating fuzzy duplicates in data warehouses. International Conference on Very Large Data Bases (VLDB); 2002. p. 586-97. https://doi.org/10.1016/ B978-155860869-6/50058-5

5. Dong X. Reference reconciliation in complex information spaces. SIGMOD International Conference on Management of Data; 2005. p. 85-96. https://doi. org/10.1145/1066157.1066168

6. Chen Z. Adaptive graphical approach to entity resolution. Joint Conference on Digital Libraries (JCDL); 2007. p. 20413. PMCid: PMC3685235.

7. Fan W. Reasoning about record matching rules. Journal Proceedings of the Very Large Data Bases (VLDB) Endowment. 2009; 2(1):407-18. https://doi. org/10.14778/1687627.1687674

8. Bansal N. Correlation clustering. Machine Learning. 2004. p. 89-113. https://doi.org/10.1023/ B:MACH.0000033116.57574.95

9. Gionis A. Similarity search in high dimensions via hashing. International Conference on Very Large Data Bases (VLDB); 1999. p. 518-29.

10. Hassanzadeh O. Framework for evaluating clustering algorithms in duplicate detection. Journal Proceedings of the Very Large Data Bases (VLDB). 2009; 2(1):1282-93. https:// doi.org/10.14778/1687627.1687771 